\title{
Modifiers of Neural Stem Cells and Aging: Pulling the Trigger of a Neurogenic Decline
}

\author{
Emmanuel Nivet ${ }^{1}$
}

Published online: 31 May 2016

(C) Springer International Publishing AG 2016

\begin{abstract}
The adult mammalian central nervous system contains resident neural stem cells able to self-renew and to generate new neurons throughout life, as well as other neural cell types. Progressive changes in adult neural stem cells accompany the aging process, which may contribute to a progressive decline in regenerative capacities, tissue degeneration, and functional impairments. For example, accelerated and pathological declines in neural stem cell functions have been associated with age-related brain diseases. Therefore, identifying and better understanding the age-associated molecular events involved in the deterioration of adult neural stem cell homeostasis is of high interest. To date, several intrinsic and extrinsic factors have been identified as putative drivers for age-related dysfunctions in brain stem cell niches. This review aims to provide a concise overview of the age-associated changes that have been reported in mammalian adult neural stem cells as well as the underlying events able to trigger those changes.
\end{abstract}

Keywords Aging $\cdot$ Neural stem cells $\cdot$ Neurogenesis · Homeostasis · Inflammation

\section{Introduction}

Neural stem cells are mitotic cells representative of early stages of brain ontogenesis, characterized by primarily

This article is part of the Topical Collection on Age-related Stem Cell Modifiers

Emmanuel Nivet

emmanuel.nivet@univ-amu.fr

1 Aix Marseille Université, CNRS, NICN UMR 7259, 13344 Marseille, France symmetric divisions that expand neural stem cell pools during early embryonic development. Under the influence of a large variety of molecular signaling cascades and cell-cell interactions, neural stem cells are progressively regionally specified [1]. Then, the mode of cell division begins to gradually shift from symmetrical to asymmetrical to generate and expand differentiated cell numbers - i.e., neurons, astrocytes, and oligodendrocytes - while maintaining a reservoir of neural stem cells. Accordingly, central nervous system (CNS) development results from multiple complex sequential events where neural stem cells are key cell types [1]. Though production, migration, and differentiation of new neurons are largely prenatal events, the adult mammalian brain maintains neurogenic activity within two discrete brain areas, namely the subventricular zone of the cortex and the subgranular zone of the dentate gyrus in the hippocampus [2]. Resident adult neural stem cells (aNSCs) in these neurogenic niches play important roles in cognitive functions such as odor discrimination and spatial learning, as well as contributing to regeneration and repair processes in response to brain insults [3]. The functions supported by adult neurogenesis are controlled at multiple levels including the number of aNSCs, whether they are in active or quiescent states, their differentiation potential, and the survival of newly generated cells [3]. Strikingly, agerelated biological alterations in the brain have been associated with a progressive decline in adult neurogenesis and correlated with functional impairments [4, 5]. Moreover, abnormalities in neurogenesis have been reported in neurodegenerative disorders for which aging is a major risk factor for disease development/progression such as in Alzheimer's, Parkinson's, and Huntington's diseases (respectively referred as $\mathrm{AD}, \mathrm{PD}$, and HD hereafter) [6]. Accordingly, aNSCs are increasingly seen as key determinants for brain functioning throughout the life span of an organism as alterations in aNSCs can contribute to the consequences of 
neurodegenerative diseases, healthy aging, and reduced CNS repair. The aging of human aNSCs is a difficult subject to study because of the scarcity and the lack of easy accessibility to reliable primary viable tissue. Thus, much of our understanding of age-associated changes in mammalian neurogenesis comes from rodent studies. A large body of evidence demonstrates that neurogenesis is regulated in a complex manner by the dynamic interplay of cell-extrinsic signals and cell-intrinsic transcriptional and epigenetic regulators. Therefore, identifying and understanding the whole picture of the age-associated molecular processes controlling temporal changes in aNSC properties/homeostasis is crucial. Indeed, a thorough knowledge of age-associated mechanisms mediating the decline of adult neurogenesis could lead to the development of therapeutic strategies aiming to counteract some of the detrimental effects linked to aging in the brain and in relation to resident aNSCs. This review aims to summarize what is currently known about the drivers and effectors of age-associated aNSC dysfunctions.

\section{Age-Associated Genomic Modifiers of Adult Neural Stem Cells}

The balance between self-renewal and differentiation of aNSCs is essential for the maintenance of a stem cell reservoir and the continuous supply of new neurons as well as other neural cell types. With aging, changes in the expression of genes critical for fine-tuning this equilibrium would likely affect aNSC homeostasis and could lead to age-associated modifications of aNSC properties such as decreased selfrenewal and/or a reduced ability to give rise to newborn neurons, which are hallmarks of aged aNSCs [4]. Accordingly, this section reviews the age-associated genetic and epigenetic mechanisms/changes that have been previously reported in aNSCs and may contribute to the decline in aNSC function during the aging process.

\section{Genetic Alterations and Aging}

\section{Nuclear and Mitochondrial DNA Instability in aNSCs}

Several studies have demonstrated that chronological aging features an increased burden of DNA damage in most cells and tissues [7, 8]. Although yet poorly studied, it is most likely that, similar to other somatic cell types, aNSCs accrue DNA damage over the lifetime of an organism, usually as the result of multiple and non-exclusive mechanisms [9]. Notably, both nuclear DNA and mitochondrial DNA (mtDNA) have demonstrated their susceptibility to be affected by aging-related processes in the human brain $[10,11]$, though determining whether these mutations affect aNSCs specifically has not been possible from whole brain samples. Nevertheless, experimental data using aNSCs from young and old mice have suggested that aging leads to a substantial mutational load within aNSCs [12]. Overall, genomic instability may result from increase DNA damage accumulation and/or a decrease in DNA damage response, which have been described as genomic changes significantly contributing to cell senescence and aging phenotypes $[9,13]$. For instance, genome instability can originate from retrotransposition, a mechanism that correlates with chronological aging as transposable elements become more active and mobile in the genomes of aging tissues $[14,15]$. Retrotransposons are mobile elements distributed in the genome that can create genomic instability through DNA double-strand breaks and point mutations, but also can impact gene regulation [16]. Interestingly, a transposable element, the Long Interspersed Nuclear Elements 1 (LINE-1), has been shown to be greatly active in the brain and particularly within the dentate gyrus region containing aNSCs [17]. In an elegant study, the Gage laboratory has demonstrated that LINE-1 retrotransposition activity, which can be regulated via SOX2-mediated repression of LINE-1, can influence the differentiation of rat neural progenitors, $[18,19]$. This becomes even more interesting when considering that SOX2 is a key transcription factor for the control of aNSC homeostasis and neurogenesis [20], and that SOX2 plays an important role in multiple mechanisms that could regulate aNSC aging [21]. More recently, SIRT6 has been also identified as an epigenetic repressor of LINE-1 (see also the "Epigenetic Modifications and Aging" section) and, interestingly, SIRT6-mediated inhibition of LINE-1 has been found to be significantly decreased in the mouse brain during the course of aging [22•]. Besides, other age-associated mechanisms can underlie the cause of age-associated DNA damage in aNSCs such as less efficient and more error-prone DNA repair systems [23]. Whichever is the mechanism responsible for DNA lesions accumulation in aNSCs during aging, this could impact on the reservoir of resident stem cells as well as on their differentiated progenies [24], though the extent of damage or the specific mutation in the genome will certainly influence the impact to aNSCs. Of interest, loss-of-function mutations have revealed aging-like phenotypes in aNSCs, highlighting the importance of a series of gene products in regulating aNSC homeostasis throughout life; including PRDM16 [25], BMI1 [26], ATM [27], SIRT1 [28], and members of the Forkhead transcription factor family O (FoxO) $[29,30]$. For example, the transcription factor FoxO3 has been associated with extreme longevity and the Brunet laboratory has highlighted the importance of this gene in the regulation of the aNSC pool in mice by showing that FoxO3 coordinates the expression of a specific program of genes that can control self-renewal, differentiation, and oxygen metabolism [30]. Thus, in the absence of FoxO3, neural stem cells are less able to self-renew and more committed to a specific lineage. Noteworthy, a common denominator for all these key proteins contributing to normal aNSC functioning is 
their respective roles in regulating oxidative stress [25, 30-32], shedding light on the fact that aging phenotypes in aNSCs could also result from the detrimental accumulation of toxic metabolites and mitochondrial dysfunctions occurring in the aging process (see also the "Mitochondria-Associated Dysfunctions and Aging" section). This supports the "free radical theory of aging" that postulates that aging and its related diseases are, at least partly, the consequence of free radical-induced damage [33, 34]. The majority of free radicals that damage biological systems are oxygen radicals and other reactive oxygen species (ROS), and mitochondria are the main source of ROS [35]. It is therefore not surprising that a higher incidence of mtDNA mutations has been shown to be associated with the aging brain as well as several age-related disorders such as AD and PD [36]. Wang and collaborators have demonstrated that mtDNA integrity is essential for aNSC function as alteration in the mtDNA repair machinery, through a knockout of 8-oxoguanine DNA glycosylase $(\operatorname{Ogg} 1)$, can hamper neuronal differentiation to the benefit of astrogliosis [37]. This is of particular interest as increased astrogliosis and decreased neurogenesis have been described in the aging brain [4]. Moreover, using genomic DNA repair defective mice, Ahlqvist et al. have demonstrated that mtDNA mutagenesis reduces the amount of quiescent aNSCs in vivo and hampers self-renewal in vitro [38], another mark of the aging brain. Altogether, these studies and many others suggest that DNA damage, including mtDNA, likely plays a causative role in aNSC aging (please refer to the accompanying article in this same issue of Current Stem Cell Reports by Espada et al. for a detailed view on DNA damage response in the context of aging).

\section{Telomere Attrition}

As another source of genomic instability, telomere shortening has been largely proposed as one of the mechanisms promoting cellular aging through the progressive loss of DNA sequences that protect chromosomes from end-to-end fusions and prevent gene erosion and so contribute to maintaining genomic integrity [39]. Notably, telomere attrition is also one of the leading causes of cellular senescence [13, 40]. Although aNSCs exhibit high telomerase activity [41], which can lengthen (and maintain) telomeres after cell division, this has been found insufficient to prevent telomere shortening during aging [42]. Interestingly, Ferron and colleagues have demonstrated that telomere shortening can alter self-renewal and differentiation properties of aNSCs [42, 43], highlighting the importance of telomere length in the maintenance of aNSC homeostasis. In addition, telomere shortening can activate $\mathrm{p} 53$, which can lead to a p53-dependent cell cycle arrest but also impair mitochondrial functions [44]. The latter feeds back to the highly probable role of mitochondrial dysfunction as a critical modifier of aNSCs during aging.

\section{Epigenetic Modifications and Aging}

\section{Chromatin and Histone Modifiers}

Epigenetic modifications essentially refer to reversible changes in DNA methylation patterns, post-translational modification of histones, and chromatin remodeling, all able to induce a change in phenotype without a change in genotype. There are multiple lines of evidence suggesting that aging is accompanied by such epigenetic changes affecting all cells and tissues, and could exert long-lasting effects throughout the life span of an organism [45]. For instance, changes in histone acetylation in the brain have been correlated with agedependent declines in brain function [46]. This indicates that epigenetic modifiers such as DNA methyl transferases, histone methyltransferases, histone demethylases, histone deacetylases, and histone acetyl transferases are probably able to induce dynamic changes during the course of aging. Of note, their activity can be under the influence of intrinsic and extrinsic cues [47]. Importantly, adult neurogenesis and therefore aNSC fate are tightly controlled by a complex interplay between various epigenetic regulators [48]. For example, members of the trithorax group (TrxG) and polycomb group (PcG) complexes, which respectively promote and inhibit gene expression via active $(\mathrm{H} 3 \mathrm{~K} 4 \mathrm{me} 3)$ or repressive (H3K27me3) marks, allow the dynamic regulation of genes that are critical for aNSC function [48]. The PcG protein BMI1 and the TrxG protein MLL1 have been described as important components implicated in age-associated aNSC self-renewal and differentiation properties [26, 49], raising the possibility that age-dependent epigenetic regulation of gene expression could play a role in decreasing neurogenesis with age. Although many other epigenetic regulatory factors have been identified as important for aNSC function and are likely to impact their neurogenic properties during aging [48, 50], for most such factors, age-dependent dynamic expression in aNSCs remains to be explored.

\section{Non-coding RNAs}

As another non-exclusive epigenetic mechanism at play during aging, non-coding RNAs can regulate gene expression in a dynamic manner that correlates with aging. For example, a network of heterochronic genes has been found to regulate temporal changes in aNSC function throughout life. Functioning as a genetic regulatory system controlling aNSC properties over time, the heterochronic microRNA let-7b is upregulated in aged aNSCs. Interestingly, it has been observed that overexpression of let-7b is sufficient to repress the expression of the high mobility group transcriptional regulator, Hmga2, which in turn increases the expression of $p 16^{\operatorname{lnk} 4 a}$ and $p 19^{4 r f}[51]$. $\mathrm{p} 16^{\text {Ink4a }}$ is a cyclin-dependent kinase inhibitor that promotes $\mathrm{Rb}$ activation, and $\mathrm{p} 19^{\mathrm{Arf}}$ promotes $\mathrm{p} 53$ protein 
stability, both slowing cell cycle progression or inducing cellular senescence [52]. As a consequence, the dynamic changes in let-7b during aging contribute to reduction in the activity of aNSC function and neurogenesis in aging animals by reducing stem cell frequency and self-renewal potential. Interestingly, this decline can be partially rescued by $p 16^{I N K 4 a}$-deficiency in aging mice [53]. Supporting further the importance of let-7b dynamic changes during the aging process, another study has revealed that let- $7 \mathrm{~b}$ can also inhibit neural stem cell proliferation and promote differentiation in embryonic brains and aNSCs through the targeting of Tlx and cyclin D1 [54]. In addition, another let-7b target, Imp1, contributes to the developmental switch changing the proliferative state of neural stem cells, from highly proliferative during fetal development to a more quiescent state in adult [55]. However, Imp1 does not seem to be expressed in aNSCs. Altogether, these studies suggest that $l e t-7 b$ plays a role in neural stem cell proliferation and differentiation across a spectrum of developmental/adult stages through the targeting of distinct key molecules, indicating that changes in let-7b may contribute to aNSC aging. More generally, these studies suggest that aNSC selfrenewal programs depend on key transcriptional regulators that can change at different stages of life. Of importance, many other microRNAs have been identified as regulators of neurogenesis such as miRs- 124, 137, and 184 [56-58] as well as the miR-106b 25 cluster that possesses a FoxO3 binding site [59]. To date, however, the impact of aging on the activity of these specific microRNAs has only been demonstrated for let-7 and miR-124 [60], though numerous microRNAs have been found differentially regulated in the brain of aged versus young mice $[60,61]$. Importantly, the impact of microRNAmediated gene regulation is not limited to healthy aging but can also correlate with neurodegenerative diseases such as $\mathrm{AD}, \mathrm{PD}$, and HD [62]. In addition, other small non-coding RNA families as well as long non-coding RNAs (lncRNAs) have been identified as important regulators of neural cells and so potentially aNSCs, and their roles in neurodegenerative disease are progressively being elucidated [62]. For example, two recent studies have identified lncRNAs that play a role in adult neurogenesis and could have functional implications in age-related neurodegenerative diseases [63, 64]. Here again, however, the dynamic expression of those non-coding RNAs remains to be investigated to establish clear links with healthy and/or pathological aging.

\section{Age-Associated Metabolic Modifiers in Adult Neural Stem Cells}

Increasing evidence suggests that energy metabolism is a critical regulator of aNSC function [65]. Accordingly, age-associated alterations/modifications in mechanisms that regulate the metabolism of aNSCs are increasingly seen as forces driving aNSC aging. For instance, aging and some aging-related brain diseases have been linked to the progressive accumulation of toxic elements within cell compartments $[33,34,66]$. The accumulation of toxic components can result from increased production of toxic metabolites as well as a reduced capacity to eliminate noxious foes. In aNSCs, it has been suggested that both mechanisms could impact the balance between selfrenewal and differentiation. More generally, several mechanisms involved in energy metabolism and energy-sensing pathways underlie the metabolic regulation of aNSC homeostasis [65, 67]. Accordingly, this section reviews the age-associated mechanisms responsible for metabolic dysregulations contributing to a neurogenic decline.

\section{Mitochondria-Associated Dysfunctions and Aging}

Studies on the aging-determining mechanisms that affect stem cell function converge to indicate the influence of the mitochondrion as a major player, and it is also the case for aNSCs [68]. Indeed, among the effectors that can induce damaging modifications in aNSCs, altered mitochondrial structure and function have been identified as possible drivers of aNSC aging [69]. Mitochondria are intracellular organelles that primarily function to generate ATP by the process of oxidative phosphorylation that leads to ROS production. During aging, a decline in mitochondrial integrity (see also "Nuclear and Mitochondrial DNA Instability in aNSCs" section) is accompanied by an abnormal accumulation of toxic by-products including ROS [33, 34]. Numerous studies suggest that control of endogenous ROS levels may play a significant role in the regulation of self-renewal and proliferation in aNSCs, as mechanisms linked to the mismanagement of ROS seem to alter aNSC function. For example, it has been demonstrated that the antioxidant enzyme SOD2, which can be regulated by the longevity-associated transcription factor FoxO3 [70], can have a protective role in mouse aNSCs [71]. Along this line, it has been shown that FoxOdeficient aNPCs have higher ROS levels and reduced self-renewal potential [29]. Furthermore, Dong and collaborators have established an experimental aging model of aNSCs, using hydroxyurea as a stress-inducer, which has confirmed the importance of ROS pathways in aNSC aging [72]. Overall, age-dependent alterations in the antioxidant capacity of the brain [73] may contribute to the neurogenic decline that comes along with age via the progressive depletion of the aNSC pool. Independent from ROS, other mitochondrial dysfunctions that have proven to be affected in the aging brain may contribute to aNSC aging, such as oxidation of mitochondrial proteins, changes in the composition of mitochondrial membranes, or defective mitophagy $[69,74,75]$. 


\section{Proteostasis-Related Alterations and Aging}

Numerous studies have revealed the importance of maintaining homeostasis of the proteome, a process called proteostasis, to ensure correct cellular function. During the aging process, however, misfolded and damaged proteins accumulate as a consequence of a functional decline in mechanisms orchestrating proteostasis such as proteolytic systems and chaperone-mediated protein folding and stability [76]. The underlying mechanisms that hinder correct proteostasis as a function of aging have been largely described elsewhere [66]. Importantly, the functional impact of impaired proteostasis has been described in multiple stem cell types [77], but little is known in aNSCs specifically. In a recent study, Moore and collaborators have indirectly highlighted the importance of proteostasis in aNSCs through a mechanism that promotes asymmetric segregation of damaged proteins in aNSCs [78••]. In this study, the authors demonstrate that aNSCs display a lateral diffusion barrier in the membrane of the endoplasmic reticulum that facilitates asymmetric segregation of damaged protein between daughter cells, allowing the maintaining of the aNSCs compartment "free of damage." With aging, however, weakening of this diffusion barrier leads to a more symmetric segregation of damaged proteins between aNSCs and their progeny, which consequently leads to progressive accumulation of misfolded proteins in aNSCs and so to reduced functionality. Another demonstration of the importance of proteostasis for aNSCs is that age is one of the main risk factors for neurodegenerative diseases associated with impaired proteostasis such as $\mathrm{AD}, \mathrm{PD}$, and $\mathrm{HD}$ [79], for which altered neurogenesis accompanied by neuronal loss has been largely described. In these diseases, the accumulation of neurotoxic proteins and/or protein aggregates are increasingly seen as triggers or aggravating factors for neurodegenerative diseases, including the aggregation of the $A \beta$ peptide and/or the accumulation of toxic $A \beta$ isoforms (e.g., $\mathrm{A} \beta 42$ ) associated with $\mathrm{AD}[80], \alpha$-synuclein associated with $\mathrm{PD}$, or toxic fragment of huntingtin in HD. Taken together, these observations support further the importance of proteostasis in aNSCs.

\section{Metabolic Sensors and Aging}

\section{Nutrient-Sensing Systems}

Nutrient sensors are key determinants in stem cell homeostasis-regulating metabolic pathways and can impact aNSC function [65]. The life-prolonging effect of calorie restriction has been largely described and such an effect is most likely mediated, at least partly, through the insulin/insulin-like growth factor 1 (IGF-1) signaling pathway (IIS). Playing a central role in the regulation of energy homeostasis via the regulation of food intake, many components of the IIS can regulate aNSC function including the PI3K/Akt-mTOR and the PI3K/Akt-FoxO pathways [65]. Supporting the role of the IIS in aNSC function and aging, it has been shown that IGF-1 can stimulate aNSC proliferation and subsequent differentiation [81], and a decrease in the level of IGF-1 has been linked to cognitive aging [82]. However, a recent study from Chaker and collaborators has offered a new perspective on the role of IGF-1 in aNSC aging [83]. Using in vivo IGF-1 receptor deletion in aNSCs, they have shown that it may not be a reduction in IGF-1 but rather a lifelong exposure to IGF-1 that causes the age-related decline of adult neurogenesis, entailing changes in IIS function and metabolic regulation [83]. Therefore, reduced levels of IGF-1 with age may indicate a reduced neurogenic activity enabling aNSCs to persist further at old age, though it cannot be ruled out that this hormone, like others (e.g., growth hormone), may exert unique effects during different stages of the life span. In addition to the IIS pathway, other nutrient-sensing systems have been investigated as putative modifiers of aNSCs that associate with aging. Among them, the mammalian target of rapamycin (mTOR) signaling pathway participates in high amino acid concentration-sensing, and the age-associated decline in the activity of the mTORC1 complex was associated with a reduced proliferation of aNSCs [84]. Additionally, AMP-dependent protein kinase (AMPK) and Sirtuins, that respectively, sense low energy states by detecting high AMP levels and high nicotinamide adenine dinucleotide $(\mathrm{NAD}+)$ levels, have been also identified as regulators of aNSC homeostasis [65]. Supporting further the importance of the coenzyme NAD+ for agerelated conditions, a recent study from Stein and Imai has demonstrated that NAD + levels decline with age in the hippocampus of mice and this decrease coincides with reduced Nampt levels and reduced differentiation and self-renewal capacities in aNSCs [85].

\section{Oxygen-Sensing Systems}

Oxygen-sensing signaling has proven to be a critical regulator of aNSC self-renewal and differentiation. More specifically, the hypoxia inducible factor- $1 \alpha$ (HIF- $1 \alpha)$, a regulator of mitochondrial function, appears to be essential for aNSC maintenance within their hypoxic niches. Indeed, HIF- $1 \alpha$ orchestrates transcriptional responses that mediate cell adaptation under hypoxic conditions. Supporting the role of oxygen-sensing signaling in aNSC, Hif- $1 \alpha$ loss-of-function experiments have demonstrated that oxygen availability is critical for correct aNSC proliferation and differentiation [86]. A deficiency in the 
activation of HIF- $1 \alpha$ downstream targets has been described in old mice [87], suggesting that impaired oxygen-sensing pathway may constitute an important factor responsible for the neurogenic decline observed in the aged brain.

\section{Age-Associated Extrinsic Modifiers of Adult Neural Stem Cells}

Beyond age-associated intracellular modifications, perturbations in aNSC homeostasis can also result from extracellular signals. Indeed, the microenvironment in which aNSCs reside is also subjected to age-associated modifications, which can impact on aNSCs via cell-cell interactions, paracrine signaling, extracellular matrix (ECM) changes, and so forth. In addition, systemic factors influence aNSC function and can contribute to age-related dysfunction. All of these can be under the influence of environmental factors including lifestyle. Therefore, this section will briefly review some of the extrinsic factors able to participate to aNSC aging.

\section{Inflammation, Systemic Factors, and Aging}

Inflammation is of paramount importance in aging and may lead to the accumulation of pro-inflammatory cytokines as the result of cellular senescence or tissue damage as well as a dysfunctional immune system. Long believed to be an immunologically privileged site, it is now well established that the brain is also subjected to immune responses and microglia, the resident macrophages of brain parenchyma, play a key role in the inflammatory response and contribute to the age-associated decline in neurogenesis [88••]. Moreover, other cell types such as astrocytes participate in the brain immune response. Although the inflammatory process is crucial for tissue homeostasis, it has been shown that chronic neuroinflammation is a major player in neurodegenerative diseases such as AD, PD, and HD [89], though whether it is cause or consequence or both is still under debate. Overall, inflammatory cytokines are key elements of complex neural-immune interactions and have been shown to impact the differentiation, proliferation, migration, and survival of aNSCs [90]. However, the effects of cytokines on aNSCs are complex as they seem to have different effects on neurogenesis, regardless whether they are antiinflammatory or pro-inflammatory cytokines. Never theless, pro-inflammatory cytokines seem to be generally more prone to inhibit neurogenesis while antiinflammatory cytokines enhance neurogenesis [91]. Adding to the complexity of our understanding on the role of cytokines on aNSC function, discrepancies exist between studies as some have described pro-neurogenic effects when others have described anti-neurogenic properties for the same cytokine. For instance, opposite effects have been described for transforming growth factor-beta (TGF $\beta$ ), an anti-inflammatory cytokine that increases in endothelial cells of the aNSC niche with age $[92,93]$. This indicates that the contribution of certain cytokines is most probably highly context- and/or dose-dependent. Paralleling the age-associated elevation of cytokines, reduced neurogenesis correlates with the elevation of other circulating molecules such as chemokines [94]. The chemokine CCL11 perhaps best exemplifies this as it was found to be elevated in both the cerebrospinal fluid and blood of aged mice, and this elevation was shown to cause reduced neurogenesis and impaired cognitive function [95*•]. The ultimate demonstration of the importance of systemic factors in aNSC aging has been brought by a series of studies showing that heterochronic parabiosis (between young and old mice) as well as the injection of young systemic factors such as GDF11 or IGF-1 can enhance the neurogenic potential of aged aNSCs [94, 95••, 96]. Of note, external factors such as exercise or caloric restriction have been described as lifestyle changes able to impact on systemic factors and, therefore, to be able to modulate aNSC function [97].

\section{Neurogenic Niche Changes and Aging}

In addition to changes in the systemic environment, agerelated changes in the cellular and acellular microenvironment (i.e., in neural stem cell niches) can impact aNSC fate. As such, astrocytes act in a paracrine manner to induce aNSCs expansion via Wnt-mediated signaling [98]. Interestingly, astrocytic Wnt3-expression decreases with age and a reduction in Wnt3 levels is associated with impaired neurogenesis [99]. Moreover, the reduction of astrocyte Wnt-mediated signaling that occurs within aging neural stem cell niches leads to downregulation of Survivin expression in aNSCs and therefore induces aNSC quiescence in the aged brain [100]. Along this line, astrocytes can release the Wnt inhibitor DKK1 that has been found to be increased with age in neural stem cell niches, which negatively regulates adult neurogenesis [101]. Taken together, these data suggest that age-associated changes in the astrocyte population within neural stem cell niches can impact aNSC function. Furthermore, aNSC function is dependent on ECM organization and composition, which can be progressively altered during aging, disease, and injury [102]. For example, changes in the mechanical properties of the ECM impact aNSC function [103]. Overall, and even though yet poorly studied, these few examples demonstrate the importance of considering age-associated changes in stem cell niches in the development of the aNSC aging phenotype. 


\section{Conclusion}

This review provides an overview of some of the main ageassociated modifications that can impact aNSC function. Noteworthy, most if not all of the age-related mechanisms that can impact aNSC function have also been described as altered in neurodegenerative diseases. This is of major relevance as identifying principles counteracting the effects of aging, acting through the mechanisms herein described, may not only lead to aNSC rejuvenation but also to functional improvements in both healthy aging and pathological situations. Obviously, no single mechanism explains all aspects of aging but, instead, it is likely that multiple non-exclusive processes contribute to a physiological decline in neurogenesis with variability between individuals. In the future, it would be important to define whether one of these mechanisms acts as a central regulator of aNSC aging and could be considered as the major trigger of a cascade of modifications, through multiple mechanisms that could accelerate physiological aging. To address such a question, lessons from the study of neurodegenerative diseases may suggest a major role for neuroinflammation, which intertwines with all of the other mechanisms and so has the potential to be a pivotal player. This remains to be demonstrated, and identifying a central regulator of aging should be a major subject of study to develop preventive/curative treatments able to counteract the effects of aging in order to maintain/restore the neurogenic capacities of the brain throughout life.

Acknowledgments This work was supported by the Fondation Plan Alzheimer.

\section{Compliance with Ethical Standards}

Conflict of Interest The author declares that there is no conflict of interest.

Human and Animal Rights and Informed Consent This article does not contain any studies with human or animal subjects performed by the author.

\section{References}

Papers of particular interest, published recently, have been highlighted as:

- Of importance

•. Of outstanding importance

1. Stiles J, Jernigan TL. The basics of brain development. Neuropsychol Rev. 2010;20(4):327-48.

2. Alvarez-Buylla A, Lim DA. For the long run: maintaining germinal niches in the adult brain. Neuron. 2004;41(5):683-6.
3. Zhao C, Deng W, Gage FH. Mechanisms and functional implications of adult neurogenesis. Cell. 2008;132(4):645-60.

4. Encinas JM, Michurina TV, Peunova N, Park JH, Tordo J, Peterson DA, et al. Division-coupled astrocytic differentiation and age-related depletion of neural stem cells in the adult hippocampus. Cell Stem Cell. 2011;8(5):566-79.

5. Maslov AY, Barone TA, Plunkett RJ, Pruitt SC. Neural stem cell detection, characterization, and age-related changes in the subventricular zone of mice. J Neurosci Off J Soc Neurosci. 2004;24(7):1726-33.

6. Winner B, Kohl Z, Gage FH. Neurodegenerative disease and adult neurogenesis. Eur J Neurosci. 2011;33(6):1139-51.

7. Kenyon CJ. The genetics of ageing. Nature. 2010;464(7288):504-12.

8. Sperka T, Wang J, Rudolph KL. DNA damage checkpoints in stem cells, ageing and cancer. Nat Rev Mol Cell Biol. 2012;13(9):579-90.

9. Signer RA, Morrison SJ. Mechanisms that regulate stem cell aging and life span. Cell Stem Cell. 2013;12(2):152-65.

10. Lu T, Pan Y, Kao SY, Li C, Kohane I, Chan J, et al. Gene regulation and DNA damage in the ageing human brain. Nature. 2004;429(6994):883-91.

11. Mecocci P, MacGarvey U, Kaufman AE, Koontz D, Shoffner JM, Wallace DC, et al. Oxidative damage to mitochondrial DNA shows marked age-dependent increases in human brain. Ann Neurol. 1993;34(4):609-16.

12. Bailey KJ, Maslov AY, Pruitt SC. Accumulation of mutations and somatic selection in aging neural stem/progenitor cells. Aging Cell. 2004;3(6):391-7.

13. Collado M, Blasco MA, Serrano M. Cellular senescence in cancer and aging. Cell. 2007;130(2):223-33.

14. Maxwell PH, Burhans WC, Curcio MJ. Retrotransposition is associated with genome instability during chronological aging. Proc Natl Acad Sci U S A. 2011;108(51):20376-81.

15. De Cecco M, Criscione SW, Peterson AL, Neretti N, Sedivy JM, Kreiling JA. Transposable elements become active and mobile in the genomes of aging mammalian somatic tissues. Aging. 2013;5(12):867-83.

16. Mita P, Boeke JD. How retrotransposons shape genome regulation. Curr Opin Genet Dev. 2016;37:90-100.

17. Coufal NG, Garcia-Perez JL, Peng GE, Yeo GW, Mu Y, Lovci MT, et al. L1 retrotransposition in human neural progenitor cells. Nature. 2009;460(7259):1127-31.

18. Muotri AR, Chu VT, Marchetto MC, Deng W, Moran JV, Gage FH. Somatic mosaicism in neuronal precursor cells mediated by L1 retrotransposition. Nature. 2005;435(7044):903-10.

19. Kuwabara T, Hsieh J, Muotri A, Yeo G, Warashina M, Lie DC, et al. Wnt-mediated activation of NeuroD1 and retro-elements during adult neurogenesis. Nat Neurosci. 2009;12(9):1097-105.

20. Ferri AL, Cavallaro M, Braida D, Di Cristofano A, Canta A, Vezzani A, et al. Sox 2 deficiency causes neurodegeneration and impaired neurogenesis in the adult mouse brain. Development. 2004;131(15):3805-19.

21. Sarlak G, Vincent B. The roles of the stem cell-controlling Sox 2 transcription factor: from neuroectoderm development to Alzheimer's Disease? Mol Neurobiol. 2015.

22. Van Meter M, Kashyap M, Rezazadeh S, Geneva AJ, Morello TD, Seluanov A, et al. SIRT6 represses LINE1 retrotransposons by ribosylating KAP1 but this repression fails with stress and age. Nature Commun. 2014;5:5011. This study confirms the importance of retrotransposition as a mechanism associated to aging. Moreover, this study highlights the importance of ageassociated dynamic changes controled by epigenetic regulators, SIRT6 in this specific case.

23. Lombard DB, Chua KF, Mostoslavsky R, Franco S, Gostissa M, Alt FW. DNA repair, genome stability, and aging. Cell. 2005;120(4):497-512. 
24. Schneider L, Pellegatta S, Favaro R, Pisati F, Roncaglia P, Testa G, et al. DNA damage in mammalian neural stem cells leads to astrocytic differentiation mediated by BMP2 signaling through JAK-STAT. Stem Cell Rep. 2013;1(2):123-38.

25. Chuikov S, Levi BP, Smith ML, Morrison SJ. Prdm16 promotes stem cell maintenance in multiple tissues, partly by regulating oxidative stress. Nat Cell Biol. 2010;12(10):999-1006.

26. Molofsky AV, Pardal R, Iwashita T, Park IK, Clarke MF, Morrison SJ. Bmi-1 dependence distinguishes neural stem cell self-renewal from progenitor proliferation. Nature. 2003;425(6961):962-7.

27. Carlessi L, De Filippis L, Lecis D, Vescovi A, Delia D. DNAdamage response, survival and differentiation in vitro of a human neural stem cell line in relation to ATM expression. Cell Death Differ. 2009;16(6):795-806.

28. Ma CY, Yao MJ, Zhai QW, Jiao JW, Yuan XB, Poo MM. SIRT1 suppresses self-renewal of adult hippocampal neural stem cells. Development. 2014;141(24):4697-709.

29. Paik JH, Ding Z, Narurkar R, Ramkissoon S, Muller F, Kamoun WS, et al. FoxOs cooperatively regulate diverse pathways governing neural stem cell homeostasis. Cell Stem Cell. 2009;5(5):540-53.

30. Renault VM, Rafalski VA, Morgan AA, Salih DA, Brett JO, Webb $\mathrm{AE}$, et al. FoxO3 regulates neural stem cell homeostasis. Cell Stem Cell. 2009;5(5):527-39.

31. Barzilai A, Rotman G, Shiloh Y. ATM deficiency and oxidative stress: a new dimension of defective response to DNA damage. DNA Repair. 2002;1(1):3-25.

32. Liu J, Cao L, Chen J, Song S, Lee IH, Quijano C, et al. Bmi1 regulates mitochondrial function and the DNA damage response pathway. Nature. 2009;459(7245):387-92.

33. Finkel T, Holbrook NJ. Oxidants, oxidative stress and the biology of ageing. Nature. 2000;408(6809):239-47.

34. Harman D. Free radical theory of aging: an update: increasing the functional life span. Ann N Y Acad Sci. 2006;1067:10-21.

35. Balaban RS, Nemoto S, Finkel T. Mitochondria, oxidants, and aging. Cell. 2005;120(4):483-95.

36. Cha MY, Kim DK, Mook-Jung I. The role of mitochondrial DNA mutation on neurodegenerative diseases. Exp Mol Med. 2015;47, e150.

37. Wang W, Esbensen Y, Kunke D, Suganthan R, Rachek L, Bjoras $\mathrm{M}$, et al. Mitochondrial DNA damage level determines neural stem cell differentiation fate. J Neurosci Off J Soc Neurosci. 2011;31(26):9746-51.

38. Ahlqvist KJ, Hamalainen RH, Yatsuga S, Uutela M, Terzioglu M, Gotz A, et al. Somatic progenitor cell vulnerability to mitochondrial DNA mutagenesis underlies progeroid phenotypes in Polg mutator mice. Cell Metab. 2012;15(1):100-9.

39. Sahin E, Depinho RA. Linking functional decline of telomeres, mitochondria and stem cells during ageing. Nature. 2010;464(7288):520-8.

40. Lopez-Otin C, Blasco MA, Partridge L, Serrano M, Kroemer G. The hallmarks of aging. Cell. 2013;153(6):1194-217.

41. Caporaso GL, Lim DA, Alvarez-Buylla A, Chao MV. Telomerase activity in the subventricular zone of adult mice. Mol Cell Neurosci. 2003;23(4):693-702.

42. Ferron SR, Marques-Torrejon MA, Mira H, Flores I, Taylor K, Blasco MA, et al. Telomere shortening in neural stem cells disrupts neuronal differentiation and neuritogenesis. J Neurosci Off J Soc Neurosci. 2009;29(46):14394-407.

43. Ferron S, Mira H, Franco S, Cano-Jaimez M, Bellmunt E, Ramirez C, et al. Telomere shortening and chromosomal instability abrogates proliferation of adult but not embryonic neural stem cells. Development. 2004;131(16):4059-70.

44. Sahin E, Colla S, Liesa M, Moslehi J, Muller FL, Guo M, et al. Telomere dysfunction induces metabolic and mitochondrial compromise. Nature. 2011;470(7334):359-65.
45. Talens RP, Christensen K, Putter H, Willemsen G, Christiansen L, Kremer D, et al. Epigenetic variation during the adult lifespan: cross-sectional and longitudinal data on monozygotic twin pairs. Aging Cell. 2012;11(4):694-703.

46. Peleg S, Sananbenesi F, Zovoilis A, Burkhardt S, Bahari-Javan S, Agis-Balboa RC, et al. Altered histone acetylation is associated with age-dependent memory impairment in mice. Science. 2010;328(5979):753-6.

47. Feil R, Fraga MF. Epigenetics and the environment: emerging patterns and implications. Nat Rev Genet. 2011;13(2):97-109.

48. Ma DK, Marchetto MC, Guo JU, Ming GL, Gage FH, Song H. Epigenetic choreographers of neurogenesis in the adult mammalian brain. Nat Neurosci. 2010;13(11):1338-44.

49. Lim DA, Huang YC, Swigut T, Mirick AL, Garcia-Verdugo JM, Wysocka J, et al. Chromatin remodelling factor Mll1 is essential for neurogenesis from postnatal neural stem cells. Nature. 2009;458(7237):529-33.

50. Pollina EA, Brunet A. Epigenetic regulation of aging stem cells. Oncogene. 2011;30(28):3105-26.

51. Nishino J, Kim I, Chada K, Morrison SJ. Hmga2 promotes neural stem cell self-renewal in young but not old mice by reducing p16Ink4a and p19Arf Expression. Cell. 2008;135(2):227-39.

52. Lowe SW, Sherr CJ. Tumor suppression by Ink4a-Arf: progress and puzzles. Curr Opin Genet Dev. 2003;13(1):77-83.

53. Molofsky AV, Slutsky SG, Joseph NM, He S, Pardal R, Krishnamurthy J, et al. Increasing p16INK4a expression decreases forebrain progenitors and neurogenesis during ageing. Nature. 2006;443(7110):448-52.

54. Zhao C, Sun G, Li S, Lang MF, Yang S, Li W, et al. MicroRNA let-7b regulates neural stem cell proliferation and differentiation by targeting nuclear receptor TLX signaling. Proc Natl Acad Sci U S A. 2010;107(5):1876-81.

55. Nishino J, Kim S, Zhu Y, Zhu H, Morrison SJ. A network of heterochronic genes including Imp1 regulates temporal changes in stem cell properties. eLife. 2013;2:e00924.

56. Cheng LC, Pastrana E, Tavazoie M, Doetsch F. miR-124 regulates adult neurogenesis in the subventricular zone stem cell niche. Nat Neurosci. 2009;12(4):399-408.

57. Liu C, Teng ZQ, Santistevan NJ, Szulwach KE, Guo W, Jin P, et al. Epigenetic regulation of miR-184 by MBD1 governs neural stem cell proliferation and differentiation. Cell Stem Cell. 2010;6(5):433-44.

58. Szulwach KE, Li X, Smrt RD, Li Y, Luo Y, Lin L, et al. Cross talk between microRNA and epigenetic regulation in adult neurogenesis. J Cell Biol. 2010;189(1):127-41.

59. Brett JO, Renault VM, Rafalski VA, Webb AE, Brunet A. The microRNA cluster miR-106b $\sim 25$ regulates adult neural stem/ progenitor cell proliferation and neuronal differentiation. Aging. 2011;3(2):108-24.

60. Inukai S, de Lencastre A, Turner M, Slack F. Novel microRNAs differentially expressed during aging in the mouse brain. PLoS One. 2012;7(7):e40028.

61. Li N, Bates DJ, An J, Terry DA, Wang E. Up-regulation of key microRNAs, and inverse down-regulation of their predicted oxidative phosphorylation target genes, during aging in mouse brain. Neurobiol Aging. 2011;32(5):944-55.

62. Szafranski K, Abraham KJ, Mekhail K. Non-coding RNA in neural function, disease, and aging. Front Genet. 2015;6:87.

63. Lin N, Chang KY, Li Z, Gates K, Rana ZA, Dang J, et al. An evolutionarily conserved long noncoding RNA TUNA controls pluripotency and neural lineage commitment. Mol Cell. 2014;53(6):1005-19.

64. Ramos AD, Diaz A, Nellore A, Delgado RN, Park KY, GonzalesRoybal G, et al. Integration of genome-wide approaches identifies lncRNAs of adult neural stem cells and their progeny in vivo. Cell Stem Cell. 2013;12(5):616-28. 
65. Rafalski VA, Brunet A. Energy metabolism in adult neural stem cell fate. Prog Neurobiol. 2011;93(2):182-203.

66. Taylor RC, Dillin A. Aging as an event of proteostasis collapse. Cold Spring Harbor perspectives in biology. 2011;3(5)

67. Ochocki JD, Simon MC. Nutrient-sensing pathways and metabolic regulation in stem cells. J Cell Biol. 2013;203(1):23-33.

68. Ahlqvist KJ, Suomalainen A, Hamalainen RH. Stem cells, mitochondria and aging. Biochim Biophys Acta. 2015;1847(11): 1380-6.

69. Stoll EA, Cheung W, Mikheev AM, Sweet IR, Bielas JH, Zhang J, et al. Aging neural progenitor cells have decreased mitochondrial content and lower oxidative metabolism. J Biol Chem. 2011;286(44):38592-601.

70. Hagenbuchner J, Ausserlechner MJ. Mitochondria and FOXO3: breath or die. Front Physiol. 2013;4:147.

71. Sakata H, Narasimhan P, Niizuma K, Maier CM, Wakai T, Chan $\mathrm{PH}$. Interleukin 6-preconditioned neural stem cells reduce ischaemic injury in stroke mice. Brain J Neurol. 2012;135(Pt 11):3298-310.

72. Dong CM, Wang XL, Wang GM, Zhang WJ, Zhu L, Gao S, et al. A stress-induced cellular aging model with postnatal neural stem cells. Cell Death Dis. 2014;5:e1116.

73. Siqueira IR, Fochesatto C, de Andrade A, Santos M, Hagen M, Bello-Klein A, et al. Total antioxidant capacity is impaired in different structures from aged rat brain. Int J Dev Neurosci Off J Int Soc Dev Neurosci. 2005;23(8):663-71.

74. Gemma C, Vila J, Bachstetter A, Bickford PC. Brain aging: models, methods, and mechanisms. In: Riddle DR, editor. Oxidative stress and the aging brain: from theory to prevention. Boca Raton (FL): Frontiers in Neuroscience; 2007.

75. Lionaki E, Markaki M, Palikaras K, Tavernarakis N. Mitochondria, autophagy and age-associated neurodegenerative diseases: new insights into a complex interplay. Biochim Biophys Acta. 2015;1847(11):1412-23.

76. Koga H, Kaushik S, Cuervo AM. Protein homeostasis and aging: the importance of exquisite quality control. Ageing Res Rev. 2011;10(2):205-15.

77. Vilchez D, Simic MS, Dillin A. Proteostasis and aging of stem cells. Trends Cell Biol. 2014;24(3):161-70.

78.• Moore DL, Pilz GA, Arauzo-Bravo MJ, Barral Y, Jessberger S. A mechanism for the segregation of age in mammalian neural stem cells. Science. 2015;349(6254):1334-8. This study demonstrates that damaged proteins progressively accumulate within aNSCs as the consequence of a reduced asymetric segregation of cellular damage with age.

79. Powers ET, Morimoto RI, Dillin A, Kelly JW, Balch WE. Biological and chemical approaches to diseases of proteostasis deficiency. Annu Rev Biochem. 2009;78:959-91.

80. He N, Jin WL, Lok KH, Wang Y, Yin M, Wang ZJ. Amyloidbeta(1-42) oligomer accelerates senescence in adult hippocampal neural stem/progenitor cells via formylpeptide receptor 2. Cell Death Dis. 2013;4:e924.

81. Aberg MA, Aberg ND, Hedbacker H, Oscarsson J, Eriksson PS, Peripheral infusion of IGF-I selectively induces neurogenesis in the adult rat hippocampus. J Neurosci Off J Soc Neurosci. 2000;20(8):2896-903.

82. Sonntag WE, Ramsey M, Carter CS. Growth hormone and insulin-like growth factor-1 (IGF-1) and their influence on cognitive aging. Ageing Res Rev. 2005;4(2):195-212.

83. Chaker Z, Aid S, Berry H, Holzenberger M. Suppression of IGF-I signals in neural stem cells enhances neurogenesis and olfactory function during aging. Aging Cell. 2015;14(5):847-56.

84. Paliouras GN, Hamilton LK, Aumont A, Joppe SE, BarnabeHeider F, Fernandes KJ. Mammalian target of rapamycin signaling is a key regulator of the transit-amplifying progenitor pool in the adult and aging forebrain. J Neurosci Off J Soc Neurosci. 2012;32(43):15012-26.
85. Stein LR, Imai S. Specific ablation of Nampt in adult neural stem cells recapitulates their functional defects during aging. EMBO J. 2014;33(12):1321-40.

86. Mazumdar J, O’Brien WT, Johnson RS, LaManna JC, Chavez JC, Klein PS, et al. O2 regulates stem cells through Wnt/beta-catenin signalling. Nat Cell Biol. 2010;12(10):1007-13.

87. Rabie T, Kunze R, Marti HH. Impaired hypoxic response in senescent mouse brain. Int J Dev Neurosci Off J Int Soc Dev Neurosci. 2011;29(6):655-61.

88.• Solano Fonseca R, Mahesula S, Apple D, Raghunathan R, Dugan A, Cardona A, et al. Neurogenic niche microglia undergo positional remodeling and progressive activation contributing to age-associated reductions in neurogenesis. Stem Cells Dev. 2016;25(7):542-55. This study demonstrates that aged-associated chronic brain inflammation that is, at least in part, mediated by the sustained activation of microglia, likely contributes to declines in aNSCs function.

89. Amor S, Peferoen LA, Vogel DY, Breur M, van der Valk P, Baker $\mathrm{D}$, et al. Inflammation in neurodegenerative diseases - an update. Immunology. 2014;142(2):151-66.

90. Kokaia Z, Martino G, Schwartz M, Lindvall O. Cross-talk between neural stem cells and immune cells: the key to better brain repair? Nat Neurosci. 2012;15(8):1078-87.

91. Breton J, Mao-Draayer Y. Impact of cytokines on neural stem/ progenitor cell fate. J Neurol Neurophysiol. 2011 S4.

92. Wachs FP, Winner B, Couillard-Despres S, Schiller T, Aigner R, Winkler $\mathrm{J}$, et al. Transforming growth factor-betal is a negative modulator of adult neurogenesis. J Neuropathol Exp Neurol. 2006;65(4):358-70.

93. Kandasamy M, Lehner B, Kraus S, Sander PR, Marschallinger J, Rivera FJ, et al. TGF-beta signalling in the adult neurogenic niche promotes stem cell quiescence as well as generation of new neurons. J Cell Mol Med. 2014;18(7):1444-59.

94. Villeda SA, Luo J, Mosher KI, Zou B, Britschgi M, Bieri G, et al. The ageing systemic milieu negatively regulates neurogenesis and cognitive function. Nature. 2011;477(7362):90-4.

95.• Katsimpardi L, Litterman NK, Schein PA, Miller CM, Loffredo FS, Wojtkiewicz GR, et al. Vascular and neurogenic rejuvenation of the aging mouse brain by young systemic factors. Science. 2014;344(6184):630-4. This sudy provides evidence that ageassociated changes in aNSCs can be reversed by circulating factors associated with youth.

96. Lichtenwalner RJ, Forbes ME, Bennett SA, Lynch CD, Sonntag WE, Riddle DR. Intracerebroventricular infusion of insulin-like growth factor-I ameliorates the age-related decline in hippocampal neurogenesis. Neuroscience. 2001;107(4):603-13.

97. Bouchard J, Villeda SA. Aging and brain rejuvenation as systemic events. J Neurochem. 2015;132(1):5-19.

98. Song H, Stevens CF, Gage FH. Astroglia induce neurogenesis from adult neural stem cells. Nature. 2002;417(6884):39-44.

99. Okamoto M, Inoue K, Iwamura H, Terashima K, Soya H, Asashima M, et al. Reduction in paracrine Wnt3 factors during aging causes impaired adult neurogenesis. FASEB J Off Publ Fed Am Soc Exp Biol. 2011;25(10):3570-82.

100. Miranda CJ, Braun L, Jiang Y, Hester ME, Zhang L, Riolo M, et al. Aging brain microenvironment decreases hippocampal neurogenesis through Wnt-mediated survivin signaling. Aging Cell. 2012;11(3):542-52.

101. Seib DR, Corsini NS, Ellwanger K, Plaas C, Mateos A, Pitzer C, et al. Loss of Dickkopf-1 restores neurogenesis in old age and counteracts cognitive decline. Cell Stem Cell. 2013;12(2):204-14.

102. Gattazzo F, Urciuolo A, Bonaldo P. Extracellular matrix: a dynamic microenvironment for stem cell niche. Biochim Biophys Acta. 2014;1840(8):2506-19.

103. Keung AJ, de Juan-Pardo EM, Schaffer DV, Kumar S. Rho GTPases mediate the mechanosensitive lineage commitment of neural stem cells. Stem Cells. 2011;29(11):1886-97. 